\title{
CONTRIBUIÇÕES E DESAFIOS PARA A GESTÃO DE DOCUMENTOS DE ARQUIVO DIGITAIS SOB A PERSPECTIVA DO INTERPARES TRUST (2012-2019)
}

\author{
CONTRIBUTIONS AND CHALLENGES OF DIGITAL \\ RECORDS MANAGEMENT FROM THE PERSPECTIVE \\ OF INTERPARES TRUST (2012-2019)
}

Natália Bruno Rabeloa

Clarissa Moreira dos Santos Schmidtb

\begin{abstract}
RESUMO
Objetivo: Objetiva-se analisar os desafios e contribuições teórico-metodológicas acerca da gestão de documentos de arquivo digitais à luz da última etapa do Projeto International Research on Permanent Authentic Records in Eletronic Systems (INTERPARES), intitulada InterPARES Trust ou ITrust (2012 - 2019). Metodologia: Para tanto, realizamos pesquisa qualitativa e bibliográfica a fim de sintetizar o panorama do Projeto InterPARES, o escopo, time de pesquisadores do ITrust, além das contribuições e desafios resultantes. Exploramos também a produção científica do interPARES e obras brasileiras ou estrangeiras que o discutam. Resultados: Tal fase do projeto foi encerrada recentemente e, por isso, ainda foi pouco divulgada e sintetizada pela literatura arquivística brasileira, embora sua contribuição seja significativa para a pesquisa sobre confiabilidade dos documentos de arquivo e dados no ambiente online, ao propor o desenvolvimento de políticas, procedimentos, padrões e legislação nesse cenário. Assim, esta pesquisa pautou-se na seguinte questão: "quais são as principais contribuições teórico-metodológicas bem como os desafios sobre a gestão de documentos de arquivo digitais apontados pelo ITrust?". Conclusões: Conclui-se a necessidade de refletir sobre aplicações de tecnologias na gestão de documentos em diversos contextos organizacionais e de ordenamento jurídico, a fim de providenciar soluções para os desafios gerados pelo cenário de crescente uso do ambiente online para estes fins.
\end{abstract}

Descritores: Arquivologia. Projeto InterPARES. InterPARES Trust. Confiabilidade. Documentos digitais.

\footnotetext{
a Mestranda em Ciência da Informação pela Universidade Federal Fluminense (UFF). Email: nataliabruno@id.uff.br

b Doutora em Ciência da Informação pela Escola de Comunicações e Artes da Universidade de São Paulo (USP). Professora da Universidade Federal Fluminense (UFF). Email: clarissaschmidt@id.uff.br
} 


\section{INTRODUÇÃO}

O projeto International Research on Permanent Authentic Records in Eletronic Systems (InterPARES) ou ITrust, de âmbito internacional, dedicou-se a pesquisar o desenvolvimento de fundamentos teóricos e metodologias para a gestão e preservação digital de documentos de arquivo autênticos a longo prazo, os quais serviram de base para a formulação de normas, manuais, políticas e modelos visando a longevidade e a manutenção da autenticidade dos documentos (DURANTI, 2015).

O documento de arquivo, de acordo com Duranti (2005, p. 7), é aquele produzido ou recebido para fins de registro de atividade ou de referência. Assim, a definição de documento de arquivo digital é equivalente à ulterior, diferenciando-se na questão do suporte.

Diante de um cenário de inovações tecnológicas e do surgimento de novas questões arquivísticas relacionadas aos documentos de arquivo digitais, o projeto InterPARES foi, até o momento, desenvolvido em quatro fases, a saber: (a) InterPARES 1 (1999 - 2001), voltado para questões relativas a bancos de dados e sistemas de gerenciamento de documentos; (b) InterPARES 2 (2002 2006), pautado na produção de documentos de arquivo digitais em sistemas interativos, dinâmicos e experimentais como resultado das atividades das artes, da ciência e do governo eletrônico; (c) InterPARES 3 (2007 - 2012), direcionado à disseminação de fundamentos teóricos e metodológicos para práticas de preservação digital; e o InterPARES Trust (2013 - 2019), objeto de estudo desta pesquisa (DURANTI, 2007, p. 579; DURANTI, 2015, p. 11; INTERNATIONAL..., s.d.).Portanto, a última fase do InterPARES explorou questões relacionadas à confiabilidade dos documentos de arquivo e de dados no ambiente online, com o intuito de desenvolver políticas, procedimentos, padrões e legislação para garantir a confiabilidade pública dos registros nesse cenário (INTERNATIONAL..., s.d.). Levando em conta o encerramento recente desta fase das investigações do grupo de pesquisa multinacional, a presente pesquisa partiu da seguinte pergunta "quais são as principais contribuições teóricometodológicas, bem como os desafios acerca da gestão de documentos de 
arquivo digitais, apontados pelo ITrust?".

Para respondê-la, traçamos um breve panorama do Projeto InterPARES, identificamos o escopo, time de pesquisadores e atribuições da equipe para, por fim, elucidar as principais contribuições e desafios do InTrust. Sendo assim, nossa investigação, de natureza qualitativa, teve como objetivo mapear as contribuições do InterPARES Trust e foi desenvolvida por meio de pesquisa bibliográfica nas bases de dados Scientific Electronic Library (SCIELO), Base de Dados em Ciência da Informação (BRAPCI), Google Acadêmico e Dialnet.

\section{PANORAMA DO PROJETO INTERPARES}

O projeto InterPARES foi iniciado em 1999 e dividido em quatro etapas até o momento, nas quais buscou-se desenvolver fundamentos teóricos acerca da preservação a longo prazo de documentos de arquivo autênticos, produzidos ou armazenados em suporte digital, a fim de embasar padrões, políticas, estratégias e planos de ação com vistas à longevidade destes documentos de arquivo digitais. Entretanto, a última fase concluída do projeto, intitulada InterPARES Trust, iniciada em 2012, refletiu sobre questões relativas aos documentos de arquivo digitais e dados confiados à internet, sugerindo como solução o desenvolvimento de redes integradas e consistentes no estabelecimento de políticas, regras, leis e procedimentos técnicos (DURANTI, 2015, p. 11).

Idealizado por Luciana Duranti e financiado e sediado pela School of Library, Archival and Information Studies (SLAIS) da University of British Columbia (UBC) após a conclusão do Projeto The preservation of integrity of electronic records (Projeto UBC) que foi desenvolvido junto ao United States Department of Defense (DoD), o Projeto InterPARES, inicialmente, contou com a participação de pesquisadores de diversos países, como Estados Unidos, Canadá, Austrália, Reino Unido, Itália, Espanha, Portugal, China e outros, além da contribuição de diversas áreas do conhecimento, Arquivologia, Ciência da Informação, Direito, História, Ciência da Computação e Engenharia. Visto isso, o Projeto serviu, ao longo de sua trajetória, como base teórica para a confecção de normas, como a Design Criteria Standards for Electronic Records 
Management Software Applications (DoD 5015.2), voltada para gestão de documentos eletrônicos na administração federal americana, e Modular Requirements for Records Systems (MoReq), para gestão de documentos eletrônicos no âmbito da União Europeia (DURANTI, 2009, p. 82-83).

Em sua primeira fase, o InterPARES 1 pautou-se nos documentos de arquivo de valor permanente, tanto analógicos quanto digitais, entretanto, atentou-se especialmente às questões sobre produção de documentos, majoritariamente textuais, em sistemas de gestão de documentos e em bases de dados. Como metodologia, utilizou das análises diplomática e arquivística e estudos de casos selecionados com base na grounded theory (DURANTI, 2005, p. 5-6). Sendo assim, o tema central foi discutido ao longo desta etapa de acordo com as seguintes temáticas: identificação de elementos que presumem a autenticidade; avaliação de documentos de arquivo eletrônicos autênticos; preservação de documentos de arquivo eletrônicos; e estruturação para desenvolvimento de políticas, estratégias e padrões para preservação a longo prazo de documentos de arquivo autênticos (INTERNATIONAL..., 2001, p. 2-3).

Posteriormente, na segunda etapa, InterPARES 2, iniciada em 2002, o projeto enfatizou o tratamento arquivístico de documentos de arquivo digitais de valor corrente em ambientes vivenciais, interativos e dinâmicos ao decorrer de atividades científicas, artísticas e de governo eletrônico. Quanto à metodologia utilizada, adotou uma combinação de métodos originários de todas as áreas do conhecimento envolvidas nesta parte da pesquisa (DURANTI, 2005, p. 5-6). Desse modo, as pesquisas focalizaram nos processos de criação e uso dos documentos nestes ambientes, tais quais: confiabilidade e autenticidade dos documentos de arquivo; recolhimento de documentos por razões legais, administrativas, sociais e culturais; preservação a longo prazo de documentos de arquivo autênticos; análise e avaliação de tecnologias para a implementação destas metodologias visando diversidade cultural e pluralismo (INTERNATIONAL..., 2006, p. 1).

Quanto à terceira fase, InterPARES 3, o foco do projeto voltou-se para o fomento à preservação a longo prazo de documentos de arquivo digitais por meio de diretrizes, por organizações públicas e privadas com recursos limitados em 
favor da manutenção da autenticidade com fundamento nos produtos das pesquisas das duas fases iniciais do Projeto, como por exemplo, as Diretrizes do Preservador (2010) e as Diretrizes do Produtor (2010). Os resultados sugeriram modelos de custo-benefício e éticos, módulos de educação e metadados de diretrizes para aplicativos (BOEL; SENGSAVANG, 2021, p. XIII). Isto é, o resultado consistiu em um conjunto de requisitos para instituições e organizações arquivísticas de pequeno e grande porte e de diferentes contextos, responsáveis pela preservação de documentos de arquivo digitais autênticos no decorrer dos resultados de suas atividades (DURANTI, 2007, p. 586).

Por fim, na quarta etapa, até então a última concluída do Projeto, o InterPARES Trust, teve como objetivo desenvolver um conjunto de teorias e metodologias para apoiar o desenvolvimento de políticas, procedimentos, normas e legislações para documentos de arquivo digitais armazenados em ambientes que possam ser acessados por internet a fim de garantir sua confiabilidade. Para tanto, foram realizadas investigações para: (a) avaliar os resultados das políticas e práticas atuais no que se refere ao tratamento arquivístico de documentos de arquivo digitais em ambiente online por instituições; (b) refletir acerca de problemáticas sobre segurança da informação e declínio de confidencialidade devido ao crescimento da produção de documentos em recursos que utilizem internet; (c) estabelecer modelos de políticas, procedimentos e práticas para criação, gerenciamento, acesso e armazenamento de documentos de arquivo digitais na internet e, especialmente, em redes sociais, computação em nuvem e tecnologias desenvolvidos para celular; (d) determinar propostas e modelos que contenham requisitos funcionais para sistemas de armazenamento e gerenciamento de documentos de arquivo digitais na internet (DURANTI; JANSEN, 2013, p. 64-65).

Complementarmente, em 2021, iniciou-se a quinta fase do InterPARES, atualmente em andamento, InterPARES Trust Al ou ITrust Al, com finalidade de projetar, desenvolver e contribuir investigações sobre viabilização do acesso à informação através do uso de Inteligência Artificial (IA). Nesse âmbito foram traçados os seguintes objetivos específicos: (i) identificar tecnologias IA que podem lidar com desafios dos arquivos; (ii) determinar benefícios e riscos do uso 
de IA em documentos de arquivo; (iii) garantir requisitos funcionais conforme a teoria arquivística no desenvolvimento de IA; (iv) validar o terceiro objetivo por meio de demonstração e aplicação por estudos de caso (INTERNATIONAL..., 2021).

\section{EQUIPE DE PESQUISA E OBJETO DE ESTUDO}

O time de pesquisadores do Projeto InterPARES Trust, composto por membros de universidades e organizações públicas e privadas em âmbito internacional e de caráter interdisciplinar, foi inicialmente separado em cinco principais grupos: América do Norte, Europa, Ásia, América Latina e organizações multinacionais (DURANTI; JANSEN, 2013, p. 67). Posteriormente, foram adicionados dois novos grupos, em 2015, Australásia e África (INTERNATIONAL..., s.d.). Além disso, foram estabelecidos cinco domínios de pesquisa a fim de organizar a produção científica do Projeto com base nesta estrutura, a saber: infraestrutura, segurança, controle, acesso e aspectos legais; bem como outros cinco grupos de domínios cruzados que foram criados na medida em que ao decorrer da pesquisa foram notadas convergências entre alguns dos cinco iniciais temas propostos, tais quais: terminologia, fontes, educação, política, e questões sociais/societais.

Sendo assim, os times supracitados desenvolveram pesquisas orientadas a objetos de estudos que se enquadram nos seus respectivos domínios, como pode ser observado no Quadro 1.

Quadro 1 - Relações entre equipes e temáticas de pesquisa.

\begin{tabular}{|l|l|}
\hline Time & Principais questões abordadas \\
\hline Nomérica do & $\begin{array}{l}\text { Arranjo e descrição nas nuvens; Cálculo da confiabilidade; Termos } \\
\text { contratuais para serviços baseados nas nuvens; Terminologia; Modelo de } \\
\text { contrato para computação nas nuvens; Documentos fílmicos e a democracia; } \\
\text { Especificações de seleção e avaliação nos ambientes digitais; Estudo } \\
\text { histórico dos serviços baseados em nuvens; Questões de privacidade no } \\
\text { governo aberto e nos dados abertos; Currículo do InterPARES Trust e o } \\
\text { mapeamento de competências arquivísticas; Questões legais no record } \\
\text { keeping nas nuvens; Metadados e requisitos de autenticidade; Modelos para } \\
\text { gestão de documentos híbridos; Políticas e confiabilidade em patentes e } \\
\text { petições; Preservação na “Era Digital”; Preservação como um serviço para a } \\
\text { confiabilidade; Classificação funcional; Seleção e avaliação em ambientes } \\
\text { nas nuvens; Mídias sociais e confiabilidade no governo; Práticas } \\
\text { padronizadas para a confiabilidade na proteção de documentos }\end{array}$ \\
\hline
\end{tabular}




\begin{tabular}{|c|c|}
\hline Europa & $\begin{array}{l}\text { Confiabilidade de documentos online; Interoperabilidade de e-services do } \\
\text { governo; Gestão de documentos de arquivos na internet; Metadados para } \\
\text { avaliação documental de websites; Modelos econômicos para o } \\
\text { armazenamento nas nuvens; Confiabilidade na infraestrutura como um } \\
\text { serviço; Esquema de metadados para descrição; Acessiblidade da } \\
\text { informação; Maturidade de governança da informação; Preservação a longo } \\
\text { prazo; Dados abertos do governo; Arquivos pessoais nas nuvens: } \\
\text { regulamento e confiabilidade; Políticas para preservação e manutenção de } \\
\text { registros digitais; Seleção e avaliação de websites; Governo aberto; } \\
\text { Fundamentos ético-sociais da preservação digital; A materialidade dos } \\
\text { documentos de arquivo e os atributos da confiabilidade; O papel do gestor } \\
\text { de documentos e da gestão de documentos no governo aberto; Modelo de } \\
\text { preservação digital; Usabilidade e interação homem-computador; } \\
\text { Percepçães dos usuários sobre documentos nato-digitais; Web Analytics na } \\
\text { avaliação de documentos }\end{array}$ \\
\hline América Latina & $\begin{array}{l}\text { Gestão de correio eletrônico; Dados abertos e governo aberto na América } \\
\text { Latina; Políticas arquivísticas institucionais; Preservação e gestão do ciclo } \\
\text { de vida dos documentos no governo digital; Documentos nas nuvens; } \\
\text { Regulação para o serviço de armazenamento das nuvens }\end{array}$ \\
\hline Ásia & $\begin{array}{l}\text { Certificação digital; Gestão de documentos empresariais nas nuvens; } \\
\text { Fatores que influenciam o acesso à informação governamental; Políticas e } \\
\text { legislação para a gestão de documentos nas nuvens; Gestão e preservação } \\
\text { nas nuvens; Cadeia de responsabilidade para a gestão de documentos nas } \\
\text { nuvens; Segurança da gestão de documentos; Certificação de confiabilidade } \\
\text { a longo prazo }\end{array}$ \\
\hline Australásia & $\begin{array}{l}\text { Repositórios obscuros como serviços; Encorajamento da criação de } \\
\text { documentos digitais confiáveis nas nuvens; Caminhos dos documentos } \\
\text { arquivísticos digitais. O impacto do acesso a documentos confiáveis } \\
\text { digitalizados; Documentos públicos com acesso distribuídos; Perspectivas } \\
\text { da confiabilidade a partir do usuário }\end{array}$ \\
\hline África & $\begin{array}{l}\text { Alinhamentos curriculares em educações de nível superior para preparar os } \\
\text { profissionais para criarem documentos de arquivo em ambientes } \\
\text { interconectados; Autenticidade dos documentos no processo de auditoria; } \\
\text { Gestão de documentos arquivísticos digitais no âmbito privado }\end{array}$ \\
\hline Transnational & $\begin{array}{l}\text { Cálculo da confiabilidade dos documentos arquivísticos; Avaliação de } \\
\text { sistemas de informação: um template para análise; Ontologia de funções } \\
\text { para sistemas arquivísticos; Inviolabilidade de arquivos globais distribuídos; } \\
\text { Classificação de segurança para documentos nas nuvens; O uso de serviços } \\
\text { nas nuvens para gestão de documentos em organizações internacionais }\end{array}$ \\
\hline
\end{tabular}

Fonte: International Research on Permanent Authentic Records in Electronic Systems Trust: ITrust (s. d.)

Dessa forma, com vistas aos objetos de estudos em que se pautaram os times de pesquisa que compõem o Projeto, cabe analisar suas contribuições para a Arquivologia e refletir acerca dos desafios impostos pelo cenário de inovação tecnológica no qual o InterPARES Trust busca apresentar soluções. 


\section{CONTRIBUIÇÕES E DESAFIOS DO INTERPARES TRUST}

Dessa forma, com vistas aos objetos de estudos em que se pautaram os times de pesquisa que compõem o Projeto, cabe analisar suas contribuições para a Arquivologia e refletir acerca dos desafios impostos pelo cenário de inovação tecnológica no qual o InterPARES Trust buscou apresentar soluções.

A produção científica do InterPARES Trust pode ser agrupada em cinco categorias: checklists, modelos, relatórios, livros e artigos científicos. De acordo com Duranti, em entrevista concedida à Indolfo e Lopes (2015, p. 14), a metodologia de construção do conhecimento perpassa pela organização semestral de simpósios com os membros do time, onde são apresentadas versões das pesquisas. Dessa forma, no website do Projeto podem ser encontradas as versões parciais e finais destes cinco tipos de publicação, demonstrando que os diferentes tipos de divulgação das pesquisas estão relacionados às necessidades profissionais urgentes dos arquivistas que acompanham o projeto, bem como a divulgação acadêmica de maneira minuciosa dos métodos e resultados produzidos nos esforços investigativos.

Segundo Boel e Sengsavang (2021, p. XIII), as pesquisas do Projeto utilizaram métodos arquivísticos e diplomáticos, Teoria Baseada em Recursos, gerenciamento de riscos, Teoria do Design, Ciência Forense Digital, Interação Homem-Computador, Leis de Telecomunicação e Teoria Organizacional e podem, majoritariamente, ser aplicados independentemente do contexto cultural, jurídico, administrativo ou social.

Dentre as publicações do ITrust, destacam-se os livros: Trusting Records in the Cloud por Luciana Duranti e Corinne Rogers, Recordkeeping in International Organizations: Archives in Transition in Digital, Networked Environments, por Jens Boel e Eng Sengsavang, Trust and Records in an Open Digital Environment, por Hrvoje Stančić.

Em Trusting Records in the Cloud foram representadas as principais investigações do ITrust sobre criação, gestão de documentos e preservação de documentos de arquivo digitais em nuvem com o objetivo de auxiliar o arquivista na implementação de tabelas de temporalidade, a compreender o papel dos 
metadados em nuvem a levar em consideração na manutenção da cadeia de custódia; e refletir acerca da avaliação, classificação, arranjo e descrição neste ambiente. Além disso, apresenta ferramentas como o modelo Preservation as a Service for Trust (PaaST), para preservação de documentos de arquivo digitais em nuvem, o modelo The TrustChain, para autenticação de documentos em blockchain e checklist para contratos providos por nuvem e artigos sobre dados governamentais abertos (DURANTI; ROGERS, 2019, p. 7; BOEL; SENGSAVANG, 2021, p. XIII).

Como uma forma de lidar com os problemas relacionados às dúvidas quanto a confiabilidade e autenticidade dos documentos de arquivo armazenados em nuvens, o modelo PaaST foi criado com o objetivo de fornecer requisitos previamente testados para a manutenção da autenticidade de documentos de arquivo criados, armazenados ou gerenciados em ambiente online, fundamentado no modelo Chain of Preservation baseado no ciclo vital e na preservação desde a criação dos documentos, desenvolvido no InterPARES 2, porém, com um novo conjunto de terminologias e conceitos importados de padrões pré-existentes, como o Model for an Open Archival Information System (OAIS). Tal é o caso de documentos transferidos do sistema do criador do documento à custódia de Cloud Service Providers, em outras palavras, organizações que oferecem o serviço de armazenamento de documentos e dados em nuvens que utilizam datacenter mega-warehouses centralizados e se expandiram devido ao aumento da largura de banda e da capacidade de armazenamento de memórias (JANSEN, 2017, p. 1-3).

Por outro lado, devido aos problemas relacionados à confiabilidade das assinaturas digitais em documentos de arquivo por, geralmente, dependerem da confiança em instituições/autoridades certificadoras, de arquivamento, armazenamento de hashes e controle de assinaturas digitais, sugere-se a implementação do TrustChain, um sistema baseado em blockchain que permite qualquer pessoa interessada ao documento de arquivo a confirmar a sua inalteração e posse de assinatura válida. Sistema este onde propõe-se a cooperação de múltiplos arquivos ou instituições interessadas, apesar de não haver empecilho técnico de uma única instituição executar os requisitos de 
software e hardware para contemplar o modelo. Entretanto, neste caso seria necessário que haja presunção de confiabilidade na instituição em questão e isto justifica a vantagem da descentralização da rede por apresentar a solução para este ponto de falha. Isto é, faz-se necessária a integração de várias instituições na rede de forma a preservarem carimbo de data e hora e servirem como testemunhas aleatórias, ou seja, as instituições participadoras, junto a mecanismos de consenso comparariam o documento que precisa de verificação de assinatura com o livro-registro na blockchain onde será buscado, por meio de um sistema de indexação, o bloco com a informação que confirmará a confiabilidade da assinatura em questão (BRALIĆ; KULEŠ; STANČIĆ, 2017, p. 89-91).

Já a obra Recordkeeping in International Organizations consiste em uma reunião de estudos já publicados em outros contextos do Projeto, porém, trabalhados em conjunto, acerca de reflexões sobre recordkeeping e seu impacto em diferentes ambientes conectados internacionalmente devido ao crescimento exponencial destas tecnologias fundamentadas em experiências relatadas em estudos de caso desenvolvidos ao decorrer do ITrust. Esta publicação conta com os capítulos, sequencialmente, nestas seguintes temáticas: reflexão sobre a interação das novas tecnologias com a educação e treinamento dos arquivistas, as normas, o acesso e a pesquisa na Arquivologia; análise da segurança dos dados de instituições europeias e discussão acerca dos desafios propostos pela aplicação de inteligência artificial e big data em arquivos; mapeamento das contribuições e desafios da computação em nuvem no contexto de produção de documentos por organizações internacionais; experiências com contratos orientados a nuvens em instituição internacional; modelização de mapa mental das funções de sistemas de arquivos; a importância da arquitetura empresarial para organizações intergovernamentais; debate sobre as políticas arquivísticas pautadas em gestão de documentos, proteção e acesso a documentos (BOEL; SENGSAVANG, 2021, p. 1-3).

Quanto ao Trust and Records in an Open Digital Environment, foram integradas pesquisas referentes à gestão de documentos em nuvem e aspectos sobre confiabilidade áreas de foco: o estado, cidadãos e forma documental a fim 
de demonstrar os problemas resultantes da gestão de documentos tecnologicamente conectada em favor do acesso. Este resultado de estudos desenvolvidos por uma equipe interdisciplinar do time europeu do ITrust focalizou em demandas específicas da União Europeia, como regulamentações relativas à privacidade e assinaturas digitais do bloco econômico. Contudo, estas pesquisas podem também ter relevância global por conter modelos econômicos de armazenamento em nuvem, interoperabilidade de metadados, confiabilidade em documentos de arquivo digitais em nuvem, estimar o impacto de implementação de tecnologias emergentes como blockchain e outros (STANČIĆ, 2021, p. 1).

Vale ressaltar que estas pesquisas foram motivadas por desafios, de acordo com Boel e Sengsavang (2021, p. XIII), identificados ao encerrar o ciclo InterPARES 3, como a ausência de fundamentos teóricos e metodológicos à luz da teoria arquivística sobre a adoção da computação em nuvem e de outros ambientes online para armazenamento e gestão de documentos em favor do desenvolvimento de redes locais, nacionais e internacionais integradas e consistentes de políticas, procedimentos e normas orientadas à preservação de documentos digitais.

\section{CONSIDERAÇÕES FINAIS}

Diante das principais contribuições teóricas e metodológicas e desafios sobre a gestão de documentos de arquivo digitais apontados pela fase mais recente concluída do Projeto InterPARES, o ITrust, apresentamos um breve panorama do Projeto InterPARES, mapeado o escopo, o time de pesquisadores e atribuições da equipe, e discutindo as principais contribuições e desafios do ITrust.

Sendo assim, o Projeto InterPARES, iniciado no ano de 1999, com o intuito de desenvolver e disseminar pesquisas sobre preservação de documentos digitais, após duas décadas deparou-se com os desafios apontados pelo ITrust referentes ao crescente uso de ambientes online ou conectado internacionalmente para gestão de documentos, recordkeeping e preservação digital. 
Para ilustrar, cabe ressaltar as particularidades da gestão de documentos em nuvem, bancos de dados e blockchain e seus respectivos aspectos sobre confiabilidade. Sobre estes exemplos, os dois primeiros tratam-se de tecnologias centralizadas, enquanto o último se trata de uma tecnologia descentralizada. Isto é, cada tipo de tecnologia tem os seus particulares desafios e contribuições a serem refletidos, como por exemplo, o único ponto de falha, apontado por Lemieux e Feng (2021, p. 1-2), que consiste apenas na confiança nos funcionários que gerem os dados em tecnologias centralizadas, o que pode comprometer a autenticidade e integridade dos dados caso a organização não contenha presunção destes aspectos. Contudo, a blockchain, de forma oposta, oferece um sistema descentralizado junto a um mecanismo de consenso que evitariam fraudes, porém, ainda necessitando de complementos de funcionalidades provenientes de outros tipos de tecnologia por não ter sido ainda completamente compreendida e, muitas das vezes, a plataforma não apresenta todas as funções necessárias para gestão de documentos e recordkeeping (LEMIEUX; FENG, 2021, p. 1-2).

Dessa forma, embora haja abordagens sobre blockchain nas publicações do InterPARES Trust, nas quais uma delas propõe um modelo de produção de documentos baseado em blockchain, seria válido testar o modelo em outros contextos organizacionais e de ordenamento jurídico, bem como a combinação desta tecnologia com outras. Na medida em que, segundo Lemieux e Feng (2021, p. 1-2), autoridades que dispõem de tecnologia centralizada, como bancos de dados, apresentaram problemas de confiabilidade dos registros em razão disto depender apenas da responsabilidade dos funcionários sobre a integridade e autenticidade dos dados armazenados e, portanto, o sistema descentralizado junto ao mecanismo de consenso da blockchain baseado em confirmação e validação de blocos encadeados contendo informações sobre transações de modo a evitar adulteração, bem como o processamento de registros imutáveis, pode solucionar o ponto de falha único identificado nas tecnologias centralizadas, apesar de ainda ser possível identificar problemas de uma tecnologia emergente em blockchain. 


\section{REFERÊNCIAS}

BOEL, Jens; SENGSAVANG, Eng. Recordkeeping in international organizations: archives in transition in digital, networked environments. Abingdon: Routledge, 2021.

BRALIĆ, Vladimir; KULEŠ, Magdalena; STANČIĆ, Hrvoje. A Model for Longterm Preservation of Digital Signature Validity: TrustChain. In: INFUTURE: INTEGRATING ICT IN SOCIETY, n. 6, 2017. Proceedings [...] Zagreb: University of Zagreb, 2017.

DURANTI, Luciana. An Overview of InterPARES 3 (2007-2012). Archives \& Social Studies. Journal of Interdisciplinary Research, v. 1, n. 1, 2007, p. 577603.

DURANTI, Luciana. Entrevista com Luciana Duranti. [Entrevista concedida a] INDOLFO, Ana Celeste; LOPES, Vera Hess. Acervo, Rio de Janeiro, v. 28, n. 2, p. 11-18, 2015.

DURANTI, Luciana; JANSEN, Adam. The InterPARES Trust Project: Trust and Digital Records in an Increasingly Networked Society. In: INFUTURE, n. 4, 2013, Zagreb. Proceedings [...] Zagreb: University of Zagreb, 2013.

DURANTI, Luciana. Projeto interpares: entrevista com Luciana Duranti [Entrevista concedida a] LACOMBE, Claudia. Ponto de Acesso, v. 3, n. 1, p. 82-92, 2009. Disponível em: https://brapci.inf.br/index.php/res/v/81836. Acesso em: 19 jun. 2021.

DURANTI, Luciana; ROGERS, Corinne. Trusting Records and Data in the Cloud. Londres: Facet, 2019.

DURANTI, Luciana. Rumo a uma teoria arquivística de preservação digital: as descobertas conceituais do projeto InterPARES. Arquivo \& Administração, v. 4, n. 1, p. 5-18, 2005.

INTERNATIONAL RESEARCH ON PERMANENT AUTHENTIC RECORDS IN ELECTRONIC SYSTEMS TRUST: ITrust, [S.d.] Disponível em: https://interparestrust.org/trust. Acesso em: 19 jun. 2021.

INTERNATIONAL RESEARCH ON PERMANENT AUTHENTIC RECORDS IN ELECTRONIC SYSTEMS TRUST AI: ITrust AI, 2021. Disponível em: https://interparestrustai.org/. Acesso em: 19 jun. 2021.

INTERNATIONAL RESEARCH ON PERMANENT AUTHENTIC RECORDS IN ELECTRONIC SYSTEMS. International Research on Permanent Authentic Records in Electronic Systems (InterPARES) 2: Experiential, Interactive and Dynamic Records. 2006. Disponível em: 
http://www.interpares.org/ip2/display_file.cfm?doc=ip2_book_introduction.pdf. Acesso em: 19 jun. 2021.

INTERNATIONAL RESEARCH ON PERMANENT AUTHENTIC RECORDS IN ELECTRONIC SYSTEMS. Long-term Preservation of Authentic Electronic Records: Findings of the InterPARES Project. 2001. Disponível em: http://www.interpares.org/book/index.htm. Acesso em: 19 jun. 2021.

JANSEN, Adam. Preservation as a Service for Trust: An InterPARES Trust Specification for Preserving Authentic Records in the Cloud. In: INTERNATIONAL CONFERENCE ON DIGITAL PRESERVATION, n. 14, 2017. Proccedings [...] Kyoto: IPRES, 2017. Disponível em: http://wwwarchive.cseas.kyoto-u.ac.jp/ipres2017.jp/wp-content/uploads/5AdamJansen.pdf. Acesso em: 19 jun. 2021.

LEMIEUX, Victoria Louise; FENG, Chen (ed.). Building Decentralized Trust: multidisciplinary perspectives on the design of blockchains and distributed ledgers. Suiça: Springer, 2021.

STANČIĆ, Hrvoje. Trust and Records in an Open Digital Environment. Abingdon: Routledge, 2021.

\title{
CONTRIBUTIONS AND CHALLENGES OF DIGITAL RECORDS MANAGEMENT FROM THE PERSPECTIVE OF INTERPARES TRUST (2012-2019)
}

\begin{abstract}
Objective: the main purpose of this article was analyse the challenges and theoretical and methodologcal fundamentals in records management developed in the last stage of the International Research on Permanent Authentic Records in Eletronic Syatems (INTERPARES), InterPARES Trust or ITrust (2012-2019). Methodology: in that way we focused on qualitative and bibliographical research that resumed the Project's scenario, its scope and researcher's team. After we reflected about the mainly contributions and challenges stresses out by ITrust. Results: As long as ITrust have ended recently, it was still poorly disseminated in the brazilian archival literature, even if the contributions of the Project maybe significant for the research about trust of digital records and the policies, procedures and legislation about this subject. Facing that situation, this investigation started with the question "What the mainly contributions of ITrust?" Conclusions: The results suggest that is necessary reflect about technologies applications for records management in different organizational and legal order contexts to provide solutions to challenges of using online environment for these purposes.
\end{abstract}

Descriptors: Archival Science. InterPARES Project. InterPARES Trust. Trust. Digital Records 


\title{
CONTRIBUCIONES Y RETOS PARA LA GESTIÓN DE DOCUMENTOS DIGITALES DESDE LA PERSPECTIVA DEL INTERPARES TRUST (2012-2019)
}

\begin{abstract}
RESUMEN
Objetivo: el objetivo es analizar los desafíos y aportes teórico-metodológicos en torno a la gestión de documentos digitales a la luz de la última etapa del Proyecto de Investigación Internacional sobre Registros Permanentes Auténticos en Sistemas Electrónicos (INTERPARES), titulado InterPARES Trust o ITrust (2012 - 2019). Metodología: Así, realizamos una investigación cualitativa y bibliográfica con el fin de sintetizar el panorama del Proyecto InterPARES, el alcance, el equipo de investigación de ITrust, además de los aportes y desafíos señalados. Por tanto, exploramos la producción científica de interPARES y trabajos brasileños o extranjeros que lo discutan. Resultados: Esta fase finalizó recientemente, razón por la cual fue poco difundida y sintetizada por la literatura archivística brasileña, aunque su contribución es significativa para la investigación sobre la confiabilidad de los documentos y datos de archivo en el entorno en línea, al proponer el desarrollo de políticas, procedimientos, normas y legislación en este escenario. Así, esta investigación se basó en la siguiente pregunta: "¿Cuáles son las principales aportaciones teórico-metodológicas así como los retos en la gestión de documentos de archivo digital señalados por ITrust?". Conclusiones: Los resultados sugirieron la necesidad de reflexionar sobre las aplicaciones de la tecnología en la gestión documental en diferentes contextos organizacionales y legales, a fin de brindar soluciones a los desafíos que genera el escenario de creciente uso del entorno online para estos fines.
\end{abstract}

Descriptores: Archivística. Proyecto InterPARES. InterPARES Trust. Confiabilidad. Documentos digitales.

Recebido em: 18.10 .2021

Aceito em: 27.12.2021 